\title{
Research on estimation of optical fiber probe gas holdup based on the adaptive weighted data fusion algorithm
}

\author{
Rui $\mathrm{Li}^{1,2,3 *}$, Ruiquan $\mathrm{Liao}^{2}$ \\ ${ }^{1}$ School of Electronics \& Information, Yangtze University, Jingzhou 434034, China \\ ${ }^{2}$ Petroleum Engineering College, Yangtze University, Wuhan 430100, China \\ ${ }^{3}$ National Demonstration Center for Experimental Electrotechnics and Electronics Education, Yangtze University, Jingzhou \\ 434034, China
}

Corresponding Author Email: liruidx@yangtzeu.edu.cn

https://doi.org/10.18280/ijht.360217

Received: 17 October 2017

Accepted: 20 February 2018

\section{Keywords:}

three-phase flow, gas holdup, fiber optic

probe, adaptive weighted

\begin{abstract}
The gas holdup of oil-gas-water three-phase flow is intimately connected with stream pattern, flow rate and tension. Regarding the deviation problem in the measurement of fiber optic probe gas holdup in oil-gas-water three-phase flow, this paper presents an evaluation method to measure the gas holdup of fiber optic probe based on data fusion. Firstly, the method applies the Grubbs criterion to eliminate the omission errors in the gas holdup data collected by a single fiber optic probe in a period of time. Secondly, it acquires the optimal value of the fiber optic probe in a certain time quantum by estimating the data collected by the fiber optic probe in batches. After obtaining the optimal value of the data collected by the four fiber optic probes in the downhole optical fiber probe array, the author adopts the self-adaptive weighted fusion algorithm, fuses data according to the optimization principle of the total minimum mean squared error and calculates the accurate value of the gas holdup of oil-gas-water three-phase flow in the wellbore in a certain time quantum. The experimental results show that this kind of method can improve the measurement accuracy of gas holdup and achieve more accurate estimate in oil-gas-water three-phase flow.
\end{abstract}

\section{INTRODUCTION}

The gas holdup is an important parameter for the well production profile evaluation and measurement. It is very important for the accurate positioning of the water production horizon and the improvement of oil and gas production. Through the measurements of the gas holdup, the downhole gas content can be obtained, which helps evaluate the production status of wells and the production structure of the oil layer [1-3]. Experimental measurement is the main method for studying gas holdup. Various methods for measuring gas holdup have been studied at home and abroad, such as: optical Doppler-reflection wave method, ultrasonic method, fast closing valve method and capacitance method, etc. They all have their own characteristics and limitations. The use of fiber-optic probes to measure the gas holdup of oil-gas-water three-phase flow is a more advanced measurement method, whose greatest advantages are light weight, non-conductivity, and convenient data processing. The multi-phase flow in oil wells is complicated. During the measurement of gas holdup, due to the influences from various factors and the accuracy of the fiber probes, there will be some deviations in the detection results. In order to accurately measure the gas holdup, afiber probe array must be used. Selecting an appropriate data fusion algorithm for fiber probe array is the key task to improve the gas hold-up accuracy [4-6].

The multi-sensor data fusion technology helps resolve data redundancy, reduce data measurement errors, and improve measurement accuracy [7-10]. Zhang Pin et al. proposed an optimized Bayesian estimation multi-sensor data fusion method by incorporating the Kalman filter, effectively solving data inconsistency and uncertainty [11-13]. Ding Lei et al. proposed a new multi-sensor data fusion method based on support vector machine regression estimation, and proved that the method is superior to the multidimensional regression analysis and artificial neural network data fusion methods through experiments [14]. Based on the data fusion method for fiber probe gas holdup estimation, firstly, the author first uses the Grobas criterion to eliminate the omission errors in the gas holdup data collected by a single fiber probe over a period of time, and divides the fiber probe collected data to obtain the optimal estimate of the fiber probe for a certain period of time, and then obtains the optimal estimated value of the data collected by the four fiber probes in the downhole fiber-optic probe array, and then fuses the data with the adaptive weighted fusion algorithm according to the optimal weight distribution principle and calculates the accurate gas holdup of the oil-gaswater three-phase flow in the wellbore.

\section{GAS HOLDUP MEASUREMENT PRINCIPLE}

The refractive index of the liquid phase is approximately 1.35 , and the refractive index of the gas phase is approximately 1. Due to the different degrees of total reflection of light in the gas phase and the liquid phase with different refractive indices, the gas holdup can be measured by a fiber probe according to this principle. As shown in Figure 1, when the optical probe is in contact with water, due to the large refractive index of the medium, total reflection will not occur at the probe, with a 
small optical power detected at the exit end, and the output at an approximately low level which use $\mathrm{v}_{\mathrm{L}}$ represent [15-17]; but when the optical probe is in contact with the gas phase, due to the small refractive index of the medium, total reflection will occur at the probe, with a large optical power detected at the exit end, and the output at an approximately high level which use $v_{H}$ represent. Therefore, the signals from the high and low levels of the emitted light can be used to determine whether the contacted medium is in the liquid phase or gas phase, so as to achieve gas holdup measurement. The fiber optic probe uses a sapphire fiber as the sensitive probe. The diameter of the sapphire fiber probe is $600 \mathrm{um}$. The probe is designed into a conical shape with a tip angle of $25-35^{\circ}$.
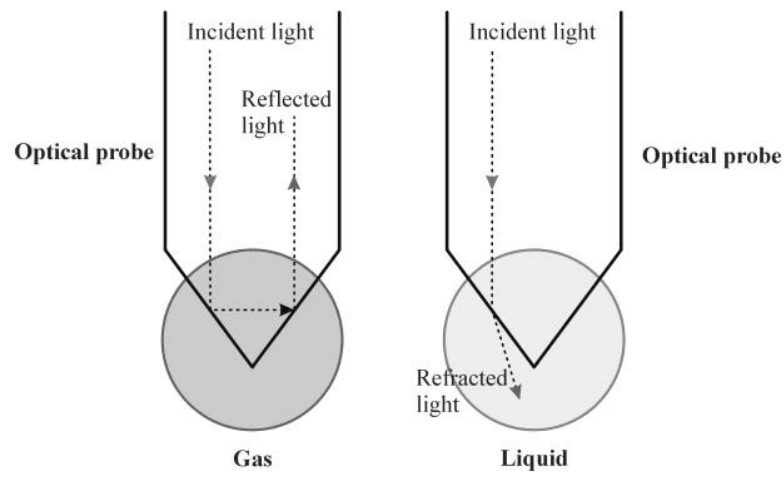

Figure 1. Working principle of the optic fiber probe

Set up threshold voltage asv $v_{S}$. A single threshold method is used in the processing of the fiber probe output signal. The threshold $v_{s}$ is used to distinguish whether the signal is in the gas or liquid phase. When the signal is greater than the thresholdv $_{\mathrm{S}}$, the fiber probe is in the gas phase; otherwise the probe is in the liquid phase.

Letv $_{\mathrm{s}}=0.3 \times\left(\mathrm{v}_{\mathrm{H}}-\mathrm{v}_{\mathrm{L}}\right)$, compare the $\mathrm{C}_{\mathrm{H}} \operatorname{andv}_{\mathrm{L}}$ withv $_{\mathrm{S}}$, then let the output pass a shaping circuit; the output waveform of the optical fiber probe is shown in Figure2. The gas holdup can be obtained based on the measured high-level time and the total measurement time.

$\alpha=\frac{1}{T} \sum_{i=1}^{n} t_{i}$

where, $\mathrm{T}$ is the total measurement time,t is the time occupied by the gas phase, $i=1,2 \ldots n$, and $\propto$ is the instantaneous gas holdup.

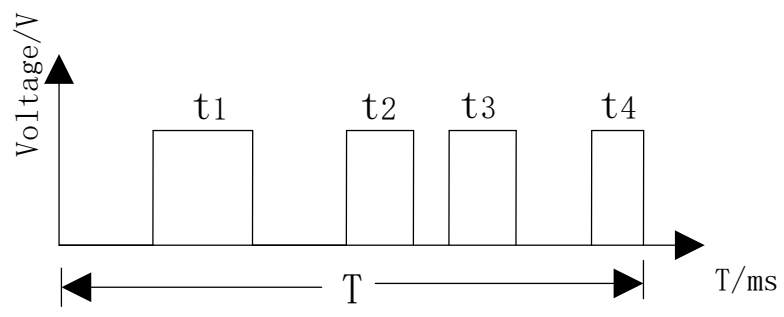

Figure 2. Fiber probe output waveform after signal processing

\section{FIBER OPTIC PROBE MEASURED GAS HOLDUP DATA FUSION SCHEME}

In order to effectively measure the average gas holdup of oil-gas-water three-phase flow in oil wells, according to the uneven distribution feature of gas holdups in oil wells, four fiber-optic probes are used to compose the fiber array structure to measure the cross-section to maximize the effective measurement range of each fiber probe. The Grubbs criterion is applied in the measurement of the local instantaneous gas holdup by a single fiber probe. In this way, the omission errors are eliminated. The optimal estimate of the optical fiber probe within a certain period of time is obtained using the batch estimation theory. Finally, the radial section of the tube is used. Considering the feature of uneven gas holdup distribution, the adaptive weighted fusion estimation method is used to process the sensor data in the fiber probe array to obtain the fusion rate of gas holdups in the well. The block diagram of the fiber probe array sensor data fusion and gas holdup estimation is shown in Figure 3.

\begin{tabular}{|c|c|c|c|c|}
\hline $\begin{array}{l}\text { Fiber Probe } 1 \\
\text { collects data }\end{array}$ & $\begin{array}{c}\text { Grobas criterion } \\
\text { pretreatment }\end{array}$ & \begin{tabular}{|c}
$\begin{array}{c}\text { Batch estimation } \\
\text { fusion }\end{array}$ \\
\end{tabular} & \multirow{4}{*}{$\begin{array}{c}\text { Adaptive } \\
\text { Weighted } \\
\text { fusion }\end{array}$} & \multirow{4}{*}{\begin{tabular}{|c} 
Gas \\
hold-rate \\
fusion \\
estimatior
\end{tabular}} \\
\hline $\begin{array}{l}\text { Fiber Probe } 2 \\
\text { collects data }\end{array}$ & $\begin{array}{c}\begin{array}{c}\text { Grobas criterion } \\
\text { pretreatment }\end{array} \\
\text {. }\end{array}$ & $\begin{array}{c}\text { Batch estimation } \\
\text { fusion }\end{array}$ & & \\
\hline $\begin{array}{l}\text { Fiber Probe } 3 \\
\text { collects data }\end{array}$ & $\begin{array}{c}\text { Grobas criterion } \\
\text { pretreatment }\end{array}$ & $\begin{array}{c}\begin{array}{c}\text { Batch estimat ion } \\
\text { fusion }\end{array} \\
\end{array}$ & & \\
\hline $\begin{array}{l}\text { Fiber Probe } 4 \\
\text { collects data }\end{array}$ & $\begin{array}{c}\text { Grobas criterion } \\
\text { pretreatment }\end{array}$ & $\begin{array}{l}\text { Batch estimation } \\
\text { fusion }\end{array}$ & & \\
\hline
\end{tabular}

Figure 3. Block diagram of optic fiber probe array sensor data fusion and gas holdup estimation

\section{FIBER PROBE ARRAY SENSOR DATA PRE- PROCESSING}

The pattern of oil-gas-water three-phase flow in oil wells is complex. The flow and volume at each sensor in the fiber-optic probe array are different in a certain period of time, resulting in certain errors in the data collected by each fiber-optic probe [18-21]. In order to eliminate the sensor data with large errors, all data collected by the fiber probe array arepre-processed using the Grubbs criterion. This paper studies the data collected by one of the fiber probes that make up the fiber probe array. The data collected during a certain period of time is represented as $i=1 \ldots n$. The arithmetic mean of the first group of $\mathrm{n}$ data is:

$T_{1}=\frac{1}{n} \sum_{i=1}^{n} x_{i}$

Residual error: $V_{i}=x_{i}-T_{1}$

Approximate error: $\sigma_{1}=\sqrt{\frac{1}{n-1} \sum_{i=1}^{n} V_{i}^{2}}$

According to the Grubbs criterion, the author uses a table look-up method to find out the critical value of the Grubbs $\operatorname{statisticg}_{0}(n, a)$, where $a$ is usually taken as 0.05 . Let $b=$ $\mathrm{g}_{0}(n, 0.05) \times \sigma_{1}$.The author compares the relationship between $\mathrm{b}$ and the residual error. If $\left|V_{i}\right|>b$, it indicates that the corresponding sensor data $x_{i}$ should be rejected; and if $\left|V_{i}\right|<b$, the corresponding sensor data $x_{i}$ should be retained. 
This method is used to analyze the $n-1$ remaining sensor data until all omission errors are removed.

\section{FIBER PROBER ARRAY SENSOR DATA FUSION ALGORITHM}

The gas holdup at a certain time in the oil wellbore is determined based on the results of multiple sensors in the fiber probe array. In order to improve the measurement accuracy of gas holdup in the wellbore, a single fiber-optic probe is firstly used to collect data during this period of time. Then the author estimates the acquired values in batches, and then fuses the multi-fiber probe sensor data.

\subsection{Batch estimation of individual fiber probe sensor data}

The author divides the single fiber probe sensor data after the omission errors are divided into two groups, calculates the arithmetic average and standard error of each group, and performs data fusion according to the batch estimation theory.

Let the number of measurement data of a single fiber probe sensor be $n$, which is divided to 2 groups. Let the number of first group of data $x_{1 i}$ be $a$, and the number of second group of data $_{2 i}$ be $b$, where $a+b=n$.

The arithmetic average of the first group of measurement data is: $T_{(1)}=\frac{1}{a} \sum_{i=1}^{a} x_{1 i}$

The corresponding standard error is:

$\sigma_{(1)}=\sqrt{\frac{1}{a-1} \sum_{i=1}^{a}\left(x_{1 i}-T_{(1)}\right)^{2}}$

The arithmetic average of the second group of measurement data is:

$T_{(2)}=\frac{1}{b} \sum_{i=1}^{b} x_{2 i}$

The corresponding standard error is:

$\sigma_{(2)}=\sqrt{\frac{1}{b-1} \sum_{i=1}^{b}\left(x_{2 i}-T_{(2)}\right)^{2}}$

According to the research ${ }^{[8-10]}$, the data fusion of the optimal estimates of a single fiber probe can be achieved by using the batch estimation theory in statistics. The optimal estimation of the data is:

$y=\frac{\sigma_{(1)}^{2} T_{(1)}+\sigma_{(2)}^{2} T_{(2)}}{\sigma_{(1)}^{2}+\sigma_{(2)}^{2}}$

The squared error:

$$
\bar{\sigma}=\frac{\sigma_{(1)}^{2} \sigma_{(2)}^{2}}{\sigma_{(1)}^{2}+\sigma_{(2)}^{2}}
$$

Using the same method, the author estimates the gas holdup and squared error for each of the remaining 3fiber probes in the fiber probe array based on the batch estimation theory. The fused value is recorded as $y_{i}$ and the squared error is recorded as $\bar{\sigma}_{i}$.

\subsection{Multi-fiber probe sensor adaptive weighted data fusion}

After batch estimation, the algorithm measures the fused value and squared error of gas holdups of single fiber-optic probe sensors. According to the principle of total minimum mean squared error, the algorithm finds the corresponding weight of the data measured by each fiber probe in an adaptive manner, so that after fusion, the gas holdup value will be optimal. With the weighting factor $w_{i}$ introduced, the fused value of the gas holdup data in the fiber probe sensor array measurement is: $\hat{y}=\sum_{i=1}^{4} w_{i} y_{i}$ where $\sum_{i=1}^{4} w_{i}=1$. So the total mean squared error is: $\hat{\sigma}^{2}=\sum_{i=1}^{4} w_{i}^{2} \bar{\sigma}_{i}^{2}$, where $\hat{\sigma}^{2}$ is a multivariate quadratic function for every weighting factor $w_{i}$. In order to minimize the total squared error, the extreme value is obtained according to the multivariate function, and only when $w_{i}=1 / \bar{\sigma}_{i}^{2} \sum_{i=1}^{4} \frac{1}{\bar{\sigma}_{i}^{2}}, \hat{\sigma}^{2}$ can be minimized. At this time, $\widehat{\sigma}_{\min }^{2}=1 / \sum_{i=1}^{4} \bar{\sigma}_{i}^{2}$.

Substitute the squared error $\bar{\sigma}_{i}^{2}$ in single fiber probe sensor measurement into the solution $w_{i}$. Finally, $w_{i}$ and $y_{i}$ are used to jointly solve the fused value $\hat{y}$ of gas holdup data.

\section{EXPERIMENTAL RESULTS AND ANALYSIS}

In order to improve the data fusion accuracy, the data collected by a single fiber-optic probe is used to eliminate the omission errors in the previous period, and then a batch estimation algorithm is used to perform fusion estimation of the measured values of the fiber probe in a certain period of time. However, the multi-phase flow in the wellbore is complex, and the final determination of gas holdup is based on the results of data fusion obtained from fiber optic arrays composed of four fiber optic probe sensors. Therefore, the data from the four fiber probe sensors are minimized based on the total mean squared error.

The optimization principle is to perform adaptive weighted fusion within the group and reduce errors at each level. In the implementation process, the accuracy of three-phase flow average cross-sectional gas hold-up rate measurement is closely related to the number of fiber-optic probes in the tube and their placement positions using fiber optic probe sensors. Figure 4shows the structure of the four fiber probes, in which the main probe is in the middle, three probes are distributed around the main probe, and the front and rear probe end faces maintain a distance of about $1 \mathrm{~mm}$ in the axial direction.

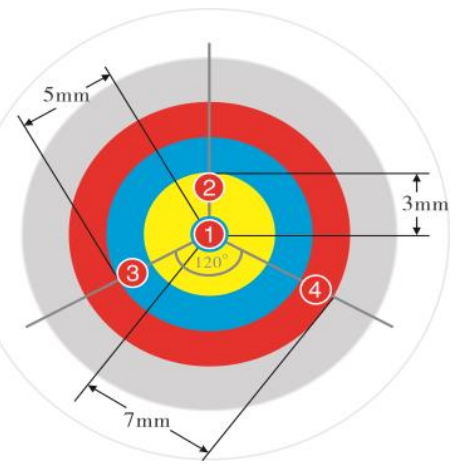

Figure 4. Fiber probe array structure 
In order to verify the effectiveness of the method, the multiphase flow laboratory of Yangtze University was used as the experimental platform. Gas holdups were measured under four different conditions: the total flow of oil and water was $30 \mathrm{~m}^{3} / \mathrm{d}$ and the air flow was $6 \mathrm{~m}^{3} / \mathrm{d}, 12 \mathrm{~m}^{3} / \mathrm{d}, 18 \mathrm{~m}^{3} / \mathrm{d}$ and $24 \mathrm{~m}^{3} / \mathrm{d}$, respectively. The experimental results are shown in Table 1. The gas rate refers to the ratio of gas volume to oil volume, and the standard gas holdup refers to the ratio of gas volume to the total oil-gas-water three-phase volume.

According to the analysis results of the gas holdup estimated by the adaptive weighted fusion and the arithmetic average in Table 1, the gas holdup estimation algorithm using self- adaptive weighted fusion under different ratios of oil-gaswater three-phase flow is superior to the cross-sectional gas holdup estimation. Compared with those obtained by the arithmetic mean estimation method, the cross-sectional gas holdup of the oil-gas-water three-phase flow can be increased by $0.05 \%-3.36 \%$, which is closer to the true value.

In the table 1, Q30G6- total oil and water flow is $30 \mathrm{~m}^{3} / \mathrm{d}$ and total gas flow is $6 \mathrm{~m}^{3} / \mathrm{d}$; Q30G12 - total oil and water flow is $30 \mathrm{~m}^{3} /$ dand total gas flow is $12 \mathrm{~m}^{3} / \mathrm{d}$; Q30G18-total oil and water flow is $30 \mathrm{~m}^{3} / \mathrm{d}$ and total gas flow is $18 \mathrm{~m}^{3} / \mathrm{d}$; Q30G24 total oil and water flow is $30 \mathrm{~m}^{3} / \mathrm{d}$ and total gas flow is $24 \mathrm{~m}^{3} / \mathrm{d}$.

Table 1. Fused cross-sectional gas holdup under different flow rates of oil-gas-water three-phase flow

\begin{tabular}{|c|c|c|c|c|c|c|c|c|c|c|c|c|}
\hline \multirow{2}{*}{$\begin{array}{l}\text { Gas } \\
\text { rate } \\
(\%)\end{array}$} & \multirow{2}{*}{$\begin{array}{l}\text { Standard } \\
\text { holdup } \\
(\%)\end{array}$} & \multirow[t]{2}{*}{ condition } & \multicolumn{2}{|c|}{$\begin{array}{l}\text { Optical fiber } \\
\text { probe1 }\end{array}$} & \multicolumn{2}{|c|}{$\begin{array}{l}\text { Optical fiber probe } \\
2\end{array}$} & \multicolumn{2}{|c|}{$\begin{array}{l}\text { Optical fiber probe } \\
3\end{array}$} & \multicolumn{2}{|c|}{$\begin{array}{c}\text { Optical fiber } \\
\text { probe } 4\end{array}$} & \multirow{2}{*}{$\begin{array}{c}\text { Arith- } \\
\text { metic } \\
\text { mean } \\
(\%)\end{array}$} & \multirow{2}{*}{$\begin{array}{c}\text { Adaptive } \\
\text { weighted } \\
\text { fused } \\
\text { value } \\
(\%)\end{array}$} \\
\hline & & & $\begin{array}{c}\text { Batch } \\
\text { estimation } \\
(\%)\end{array}$ & $\begin{array}{l}\text { Squared } \\
\text { error }\end{array}$ & $\begin{array}{l}\text { Batch } \\
\text { estimation } \\
(\%)\end{array}$ & $\begin{array}{l}\text { Squared } \\
\text { error }\end{array}$ & $\begin{array}{c}\text { Batch } \\
\text { estimation } \\
(\%)\end{array}$ & $\begin{array}{l}\text { Squared } \\
\text { error }\end{array}$ & $\begin{array}{c}\text { Batch } \\
\text { estimation } \\
(\%)\end{array}$ & $\begin{array}{l}\text { Squared } \\
\text { error }\end{array}$ & & \\
\hline 20 & 16.7 & Q30G6 & 5.43 & 0.53 & 3.65 & 0.09 & \multicolumn{2}{|c|}{4.060 .05} & \multicolumn{2}{|c|}{$5.87 \quad 0.54$} & 4.05 & 4.10 \\
\hline 40 & 28.6 & Q30G12 & 9.48 & 0.33 & 8.30 & 0.01 & \multicolumn{2}{|c|}{16.340 .26} & \multicolumn{2}{|c|}{13.400 .51} & 8.34 & 8.77 \\
\hline 60 & 37.5 & Q30G18 & 16.79 & 0.22 & 15.83 & 1.10 & 25.37 & 0.38 & \multicolumn{2}{|c|}{22.151 .16} & 16.36 & 19.72 \\
\hline 80 & 44.4 & Q30G24 & 24.28 & 0.02 & 31.04 & 0.02 & 34.95 & 0.52 & \multicolumn{2}{|c|}{28.090 .10} & 26.49 & 28.00 \\
\hline
\end{tabular}

\section{CONCLUSIONS}

In this paper, an adaptive weighted fusion algorithm is proposed to estimate the gas holdup in oil-gas-water threephase flow. The algorithm first uses the Grubbs criterion to eliminate the data with large errors collected by a single fiberoptic probe sensor over a period of time, and then it estimates the data of the fiber-optic probe sensor in batches to obtain the best estimate of the fiber-optic probe during this period of time, and obtains the best estimate of the gas holdups of the remaining three fiber-optic probe sensors. According to the principle of total minimum mean squared error, four fiber probe sensor data are adaptively weighted and fused to calculate the accurate cross-section gas holdup of the oil-gaswater three-phase flow in the wellbore at this time. The experimental results show that compared with the arithmetic average method, the adaptive weighted fusion algorithm can more effectively pre-process the anomaly data in the case of complex three-phase flow when being used to estimate the gas holdup in the oil-gas-water three-phase flow.

\section{REFERENCES}

[1] Yu L, Du SX, Li YW, Liu X, Zhang H. (2014). Study on measurement method of gas holdup of oil-gas-water three phase flow based on sapphire optical fiber probe. Well logging technology 38(2): 139-143. https://doi.org/10.3969/j.issn.1004-1338.2014.02.003

[2] Zhang RC, Yang RC. Shen Y. (1997). Measurement of void fraction by an advanced single-fiber optical probe. Journal of Engineering Thermophysics 18(1): 99-102. https://doi.org/10.1007/BF02951625

[3] Liang YM, Xu LH. (2009). Ranging sensors measurement adaptive weighted fusion online.ComputerMeasurement\&Control 17(7): 1447-1449. https://doi.org/10.16526/j.cnki.11-4762/tp.2009.07.065

[4] Mudde RF, Saito T. (2001). Hydrodynamicalsimilarities between bubble column and bubbly pipe flow. Journal of fluid mechanics, 437: 2030228. https://doi.org/10.1017/S0022112001004335

[5] Schmitt A, Homann K, Loth R. (1995). A transputerbased measuring system for decentralized signal processing applied to two phase flow. Rev. Sci. Instrum. 66(10), 5045-5049. https://doi.org/10.1063/1.1146128.

[6] Luther S, Rensen J, Guet S. (2004). Bubble aspect ratio and velocity measurements using a four-point fiberoptical probe. Experiments in Fluids 36: 326-33. https://doi.org/10.1007/s00348-003-0725-7

[7] Cartellier A. (1998). Simultaneous void fraction measurement, bubble velocity, and size estimate using a single optical probe in gas-liquid two-phase flows. Review of Scientific Instruments 63(11): 5442-5453. https://doi.org/10.1063/1.1143416.

[8] Xue J, Al-Dahhan M, Dudukovic MP, Mudde RF. (2003). Bubble dynamics measurements using four-point optical probe. Canadian Journal of Chemical Engineering 81: 375-81. https://doi.org/10.1002/cjce.5450810306

[9] Morris D, Teyssedou A, Lapierre J, Tapucu A, (1987). Optical fiber probe to measure local void fraction profiles. Appl. Opt 26(21): 4660-4664. https://doi.org/AO.26.004660.

[10] Guet S, Fortunati RV, Mudde RF, Ooms G. (2003). Bubble velocity and size measurement with a four-point optical fiber probe. Particle \& Particle Systems Characterization 20: 219-230. https://doi.org/10.1002/ppsc.200390028.

[11] Mizushima Y, Sakamoto A, Saito T. (2013). Measurement technique of bubble velocity and diameter in a bubble column via single-tip optical-fiber probing with judgment of the pierced position and angle. Chemical Engineering Science 100(2): 98-104. https://doi.org/10.1016/j.ces.2013.01.046

[12] Xue J, Al-Dahhan M, Dudukovic MP, Mudde RF. (2008) Bubble velocity, size, and interfacial area measurements in a bubble column by four-point optical probe. Aiche Journal 54(2): 350-363. https://doi.org/10.1002/aic.11386 
[13] Hosokawa S, Matsumoto T, Tomiyama A. (2013). Measurement of bubble velocity using spatial filter velocimetry. Experiments in Fluids 54(6): 1-12. https://doi.org/10.1007/s00348-013-1538-y

[14] Al-Oufi FM, Rielly CD, Cumming IW. (2011). An experimental study of gas void fraction in dilute alcohol solutions in annular gap bubble columns using a fourpoint conductivity probe. Chemical Engineering Science 66(23): $5739-5748$ http://dx.doi.org/10.1016/j.ces.2011.03.061

[15] Vries JD, Luther S, Lohse D. (2002). Induced bubble shape oscillations and their impact on the rise velocity. The European Physical Journal B 29(3): 503-509. https://doi.org/10.1140/epjb/e2002-00332-5

[16] Rastello M, Marié J, Lance M. (2011). Drag and lift forces on clean spherical and ellipsoidal bubbles in a solid-body rotating flow. Journal of Fluid Mechanics 682(3): 434-459. https://doi.org/10.1017/jfm.2011.240

[17] Dan VN. (2012). Single optical fiber probe for optogenetics. Proceedings of SPIE 8225(1): 34-42. https://doi.org/10.1117/12.907453
[18] Ojha A. (2018). Investigation of Local Gas Holdup and Bubble Dynamics using Four-Point Optical Probe Technique in a Split-Cylinder Airlift Reactor. International Journal of Multiphase Flow 102(2018): 115 .

https://doi.org/10.1016/j.ijmultiphaseflow.2017.12.001

[19] Al-Masry WA. (2015). Effect of liquid volume in the gas-separator on the hydrodynamics of airlift reactors. Journal of Chemical Technology \& Biotechnology 74(10): 931-936. https://doi.org/10.1002/(SICI)10974660(199910)74:10<931:AID-JCTB127>3.0.CO; 2-Z

[20] Bello RA, Robinson CW, young MM. (1985). Gas holdup and overall volumetric oxygen transfer coefficient in airlift contactors. Biotechnology \& Bioengineering 27(3): 369-81. https://doi.org/10.1002/bit.260270323

[21] Al-Masry WA, Dukkan AR. (1997). The role of gas disengagement and surface active agents on hydrodynamic and mass transfer characteristics of airlift reactors. Chemical Engineering 65(3): 263-271. https://doi.org/10.1016/S1385-8947(97)00033-8 